veracity for the public and for health officials. Alternatively, failure to adequately address these domains may erode of the public's trust in public health recommendations.

Acknowledgments. The author's affiliation center belongs to the Integrative Biosciences Center, Wayne State University, Detroit, Michigan. The funders had no role in the decision to publish or preparation of the manuscript.

Financial support. No financial support was provided relevant to this article.

Conflicts of interest. The author reports no conflicts of interest relevant to this article

\section{References}

1. Brooks JT, Beezhold DH, Noti JD, et al. Maximizing fit for cloth and medical procedure masks to improve performance and reduce SARS-CoV-2 transmission and exposure, 2021. Morb Mortal Wkly Rep 2021;70:254-257.

2. Brouwers MC, Kerkvliet K, Spithoff K. The AGREE reporting checklist: a tool to improve reporting of clinical practice guidelines. BMJ 2016;352: i1152.

3. Shekelle PG, Woolf SH, Eccles M, Grimshaw J. Clinical guidelines: developing guidelines. BMJ 1999;318:593-596.

4. Chu DK, Akl EA, Duda S, Solo K, Yaacoub S, Schünemann HJ; COVID-19 Systematic Urgent Review Group Effort (SURGE) study authors. Physical distancing, face masks, and eye protection to prevent person-to-person transmission of SARS-CoV-2 and COVID-19: a systematic review and meta-analysis. Lancet 2020;395:1973-1987.

5. Murad MH. Clinical practice guidelines: a primer on development and dissemination. Mayo Clin Proc 2017;92:423-433.

6. Frieden TR, Cash-Goldwasser S. Of masks and methods. Ann Intern Med 2021. doi: 10.7326/M20-7499.

7. Nanda A, Hung I, Kwong A, Man VC, Roy P, Davies L, Douek M. Efficacy of surgical masks or cloth masks in the prevention of viral transmission: systematic review, meta-analysis, and proposal for future trial. J Evid Based Med 2021. doi: 10.1111/jebm.12424.

8. Bundgaard H, Bundgaard JS, Raaschou-Pedersen DET, et al. Effectiveness of adding a mask recommendation to other public health measures to prevent SARS-CoV-2 infection in Danish mask wearers: a randomized controlled trial. Ann Intern Med 2020:M20-6817.

9. American Academy of Pediatrics Steering Committee on Quality Improvement and Management. Classifying recommendations for clinical practice guidelines. Pediatrics 2004;114:874-877.

10. Ioannidis JP. Why most clinical research is not useful. PLoS Med 2016;13: e1002049.

11. Does the prolonged use of face masks by HCW interfere with the respiratory system by inducing oxidative stress and blood oxygen/carbon dioxide imbalance? National Health Library and Knowledge Service website. https:// hselibrary.ie/does-the-prolonged-use-of-face-masks-by-hcws-interfere-withthe-respiratory-system-by-inducing-oxidative-stress-and-blood-oxygen-carbondioxide-imbalance/. Published December10, 2020. Accessed February 18, 2021.

12. Rosner E. Adverse effects of prolonged mask use among healthcare professionals during COVID-19. J Infect Dis Epidemiol 2020;6:130.

13. Samannan R, Holt G, Calderon-Candelario R, et al. Effect of face masks on gas exchange in healthy persons and patients with chronic obstructive pulmonary disease. Ann Am Thorac Soc 2021;18:541-544.

14. Scheid JL, Lupien SP, Ford GS, West SL. Commentary: physiological and psychological impact of face mask usage during the COVID-19 pandemic. Int J Environ Res Public Health 2020;17:6655.

15. Taylor S, Asmundson GJG. Negative attitudes about facemasks during the COVID-19 pandemic: the dual importance of perceived ineffectiveness and psychological reactance. PLoS One 2021;16(2):e0246317.

16. Brouwers MC, Kho ME, Browman GP, et al. AGREE Next Steps Consortium. AGREE II: advancing guideline development, reporting and evaluation in health care. CMAJ 2010;182:E839-E842.

\title{
"Original antigenic sin": A potential threat beyond the development of booster vaccination against novel SARS-CoV-2 variants
}

\author{
Maryam Noori $\mathrm{MD}^{1}$, Seyed Aria Nejadghaderi $\mathrm{MD}^{2,3}$ and Nima Rezaei MD, PhD ${ }^{4,5,6}$ (1) \\ ${ }^{1}$ Student Research Committee, School of Medicine, Iran University of Medical Sciences, Tehran, Iran, ${ }^{2}$ Systematic Review and Meta-analysis Expert Group \\ (SRMEG), Universal Scientific Education and Research Network (USERN), Tehran, Iran, ${ }^{3}$ School of Medicine, Shahid Beheshti University of Medical Sciences, \\ Tehran, Iran, ${ }^{4}$ Research Center for Immunodeficiencies, Children's Medical Center, Tehran University of Medical Sciences, Tehran, Iran, ${ }^{5}$ Department of \\ Immunology, School of Medicine, Tehran University of Medical Sciences, Tehran, Iran and ${ }^{6}$ Network of Immunity in Infection, Malignancy and Autoimmunity \\ (NIIMA), Universal Scientific Education and Research Network (USERN), Tehran, Iran
}

To the Editor-Recently, concern has increased over the emergence of novel severe acute respiratory syndrome coronavirus 2 (SARS-CoV-2) variants, which are spreading rapidly across the globe. These variants of concern (B.1.1.7, B.1.351, P.1, and B.1.427/429) have been initially reported in the United Kingdom, South Africa, Brazil, and California, respectively. ${ }^{1}$ All of the currently available vaccines that have received emergency use authorization, such as Johnson \& Johnson, Moderna, and Pfizer/BioNTech, are based on the Wuhan-originated virus.

Author for correspondence: Prof Nima Rezaei, E-mail: rezaei_nima@tums.ac.ir or rezaei_nima@yahoo.com

Cite this article: Noori M, Nejadghaderi SA, and Rezaei N. (2022). “Original antigenic sin": A potential threat beyond the development of booster vaccination against novel SARS-CoV-2 variants. Infection Control \& Hospital Epidemiology, 43: 1091-1092, https://doi.org/10.1017/ice.2021.199
Regarding the novel variants, the accumulation of multiple mutations in the spike protein, which is the target for neutralizing antibodies, has challenged the efficacy of these vaccines. Several previous laboratory-based studies have reported that the neutralizing activity of sera obtained from individuals who were vaccinated is lower against novel SARS-CoV-2 variants, ${ }^{2-5}$ highlighting the need for developing a booster vaccination containing new mutations of the virus.

A phenomenon called "original antigenic sin" (OAS) was firstly proposed by Francis ${ }^{6}$ in 1960. This phenomenon occurs in the second exposure of the immune system to a similar pathogen to which it has previously been exposed. In this situation, the immune system progresses to the memory response, generating crossreactive antibodies that may not be effective against the new pathogen. ${ }^{7}$ In addition, it has been speculated that overproduction 
of memory B cells could compromise the activation of naïve B cells capable of producing efficient and novel antibodies. ${ }^{8}$ In this way, OAS can trigger immune evasion of the emerging variants in those who had been affected by or vaccinated against former versions of the pathogen. In the context of coronaviruses, cross-neutralization is a rare event, but cross-reactivity in antibody binding to spike protein is common in SARS-CoV-2 and SARS-CoV. ${ }^{9}$ Furthermore, some degrees of cross-reactivity have also been demonstrated between seasonal coronaviruses and SARS-CoV-2. ${ }^{10}$ Aydillo et al ${ }^{11}$ reported a strong back-boosting of antibodies in SARS-CoV-2infected patients previously infected with human $\beta$-coronaviruses. Interestingly, a negative correlation was observed between pre-exposure to human $\beta$-coronaviruses and induction of antibodies against SARS-CoV-2, mentioning the reduction of de novo humoral immune response and occurrence of OAS in patients with preexisting immunity against related coronaviruses. ${ }^{11}$

The impact of OAS in developing vaccines is of paramount interest. The hypothesis of antigenic distance was proposed to explain how the efficacy of vaccines could be influenced by the difference or relatedness of prior vaccinations. This hypothesis is substantially evident in the case of dengue fever-related vaccine research. Once an individual is immunized against a dengue virus variant, the booster shot for the second variant is unlikely to be successful because it triggers only the original neutralizing antibodies rather than effective antibodies for the new variant. ${ }^{12}$ This scenario also applied to the human papillomavirus Gardasil 9 vaccine. The vaccine contained 4 antigens presented in the original Gardasil in addition to 5 novel antigens. Individuals who had been vaccinated previously by original Gardasil exhibited lower levels of antibodies against 5 new antigens compared with those who had been never vaccinated for human papillomavirus. ${ }^{13}$ In the context of influenza infection, Choi et $\mathrm{al}^{14}$ noticed that following the vaccination program against the 2009 pandemic $\mathrm{H} 1 \mathrm{~N} 1$ influenza, individuals who had already been given a seasonal influenza virus vaccination developed lower antibody response than those who had never been vaccinated against influenza virus. Therefore, OAS can leave individuals with limited and imprinted memory immune response, and booster vaccination containing novel versions of the pathogen may not provide as much protection.

Given the cross-reactivity feature of antibodies against SARS-CoV-2 and other $\beta$ coronaviruses, ${ }^{10}$ the occurrence of OAS for initial and subsequent variants of SARS-CoV-2 would not be unexpected. On the other hand, when it comes to the incidence of ongoing mutations, booster immunization may become a necessary countermeasure for combating the novel resistant variants to current vaccines. If OAS is feasible in the case of SARS-CoV-2 and its emerging variants, the effectiveness of the booster dose will somehow be questioned. Clinical trial NCT04785144 is recruiting to assess the immunogenicity of the mRNA-1273.351 vaccine, which has recently manufactured for immunization toward the novel South African variant of SARS-CoV-2. To evaluate the efficacy of this booster dose on those who have received 2 vaccinations of mRNA-1273, the trial has been designed in 2 arms. The first arm evaluates the administration of a booster dose containing mRNA-1273.351 solely, whereas the second arm contains both mRNA-1273.351 and mRNA-1273 in equal proportions. ${ }^{15}$ The consequences of this trial could shed light on how OAS may alter the effectiveness of booster vaccination for novel SARS-CoV-2 variants. Eventually, further well-designed animal or human studies on a different type of vaccines are required to evaluate the efficacy of booster immunization over the potential threat of OAS in SARS-CoV-2 vaccines.

\section{Acknowledgments.}

Financial support. No financial support was provided relevant to this article.

Conflicts of interest. All authors report no conflicts of interest relevant to this article.

\section{References}

1. Abdool Karim SS, de Oliveira T. New SARS-CoV-2 variants-clinical, public health, and vaccine implications. N Engl J Med 2021. doi: 10.1056/ NEJMc2100362.

2. Liu Y, Liu J, Xia H, et al. Neutralizing activity of BNT162b2-elicited serum. N Engl J Med 2021. doi: 10.1056/NEJMc2102017.

3. Shen X, Tang H, Pajon R, et al. Neutralization of SARS-CoV-2 variants B.1.429 and B.1.351. N Engl J Med 2021.

4. Wang G-L, Wang Z-Y, Duan L-J, et al. Susceptibility of circulating SARS- CoV-2 variants to neutralization. N Engl J Med 2021. doi: 10. 1056/NEJMc2103022.

5. Wang P, Nair MS, Liu L, et al. Antibody resistance of SARS-CoV-2 variants B.1.351 and B.1.1.7. Nature 2021. doi: 10.1038/s41586-021-03398-2.

6. Francis T. On the doctrine of original antigenic sin. Proc Am Philosoph Soc 1960;104:572-578.

7. Vatti A, Monsalve DM, Pacheco Y, Chang C, Anaya J-M, Gershwin ME. Original antigenic sin: a comprehensive review. J Autoimmunity 2017;83: $12-21$.

8. Kim JH, Skountzou I, Compans R, Jacob J. Original antigenic sin responses to influenza viruses. J Immunol (Baltimore) 2009;183:3294-3301.

9. Lv H, Wu NC, Tsang OT-Y, et al. Cross-reactive antibody response between SARS-CoV-2 and SARS-CoV infections. Cell Rep 2020;31:107725.

10. Shrock E, Fujimura E, Kula T, et al. Viral epitope profiling of COVID-19 patients reveals cross-reactivity and correlates of severity. Science 2020; 370(6520):eabd4250.

11. Aydillo T, Rombauts A, Stadlbauer D, et al. Antibody immunological imprinting on COVID-19 patients 2020. doi: 10.1101/2020.10.14.20212662.

12. Midgley CM, Bajwa-Joseph M, Vasanawathana S, et al. An in-depth analysis of original antigenic sin in dengue virus infection. J Virol 2011;85:410-421.

13. Chakradhar S. Updated, augmented vaccines compete with original antigenic sin. Nat Med 2015;21:540-541.

14. Choi YS, Baek YH, Kang W, et al. Reduced antibody responses to the pandemic (H1N1) 2009 vaccine after recent seasonal influenza vaccination. Clin Vacc Immunol 2011;18:1519-1523.

15. Safety and immunogenicity study of a SARS-CoV-2 (COVID-19) variant vaccine (mRNA-1273.351) in naïve and previously vaccinated adults 2021. Clinical Trials.gov website. https://clinicaltrials.gov/ct2/ show/NCT04785144. 